\title{
Introduction to HICSS-54 Minitrack on The Technical, Socio-Economic and Ethical Aspects of AI
}

\author{
Yibai Li \\ University of Scranton \\ yibai.li@scranton.edu
}

\author{
Xuefei (Nancy) Deng \\ California State University, \\ Dominguez Hills \\ ndeng@csudh.edu
}

\author{
Yichuan Wang \\ The University of Sheffield \\ yichuan.wang@sheffield.ac.uk
}

\section{Introduction}

Augmented Intelligence is a new perspective to look at artificial intelligence (AI), social computing, machine learning, big data, data mining, and related areas. It describes a system that consists of both artificial intelligence agents and human agents. Compared to the traditional AI whose ultimate goal is to replicate human intelligence [1], Augmented Intelligence aims to augment human intelligence, which includes not only the cognitive intelligence, but also emotional intelligence, social intelligence, physical intelligence and so on. It has a clear emphasis that humanity, not machines, is the core of this scientific inquiry. In recent years, more and more practitioners and researchers start to accept this more holistic view of AI [2] and to demonstrate that the ultimate goal of AI is not to replace human but to augment human. Augmented Intelligence describes a hybrid system not only the artificial intelligence agents, but also the human agents, therefore opening up new research opportunities not only for researchers in technical areas such as artificial intelligence, machine learning, big data, and data mining, but also for researchers in behavioral science, social science, organization science, human-computer interaction and many other areas

\subsection{The Technical Aspect of AI}

The technological advancements in computer science and information systems have presented $\mathrm{AI}$ as a new type of aid to human workers. AI can automate simple, repetitive tasks and allow human workers to work on creative tasks. In the business setting, by applying machine learning to natural language processing (NLP), AI can help its human counterparts improve the workflows in sales and customer sales management by analyzing the tone of voice and verbiage used on a call. The International Data Corporation (IDC) forecasts that AI will unleash unprecedented levels of workplace productivity, boost global revenues by $\$ 121$ billion over the next five years and generate more jobs than it will replace. In healthcare, AI assists radiologists with image interpretation by bringing the relevant information out of the electronic medical record and presenting it to them in a meaningful way to better inform their clinical judgment [3]. AI also facilitates innovations and the creation of intellectual products. For example, IBM Watson helped musician Alex Da Kid to create a popular song named "Not Easy" by uncovering and visualizing the most pervasive themes in people's deep emotions hidden past five years' culture data [4].

\subsection{The Socio-Economic Aspect of AI}

In today's digital economy, business practitioners are increasing scrutiny on socio-economic aspects of $\mathrm{AI}$ and exploring new opportunities through innovation spillovers. For instance, AI, used in combination with experienced farmers, could prevent unpredictability of weather or soil conditions, thereby reducing farmers' costs and boosting profits. In the financial sector, AI robot-enabled service helps consultants to complete repetitive tasks, allowing them to concentrate on more judgement-based proceeding and customer service. As the needs for social computing, continues to grow, researchers should continue to study how AI can drive the digital economy while at the same time satisfying human's needs by offering solutions to societal issues.

AI is also enhanced by a novel computing paradigm [5], the social computing. Social computing artifacts such as social media, blog, social bookmarking, and wiki generates enormous amount of data, the "big data," which enhances the human intelligence in acquiring, assimilating, and/or sharing (i.e., storage/retrieval, transmission features) social information. It also provides valuable training data set for the artificial intelligence agents.

AI will continue to pervade people's everyday work life and personal life. In the workplace, people will see more and more AI software programs and AI-enabled robots as their teammates. In people's 
personal life, AI assistants such as Siri, Amazon Echo \& Alexa, Google assistant, AI-enabled smart home are becoming a significant member of people's family. Human-AI collaboration and trust are important research topics in these contexts.

\subsection{The Ethical Aspect of AI}

Ethical concerns have arisen in the development and deployment of artificial intelligence systems. Computer and data scientists have or will encounter ethical issues such as biases in the building of machine learning models and stereotypes in the development of robots. For example, how can we ensure if a training data for a machine learning model is unbiased? What are the defining scopes, measurements, and prevention strategies of biases during the development and deployment of an AI system? How can human values be imported and accounted for in a fair manner in the design of AI systems?

Value sensitive design is a value-oriented design methodology commonly adopted in human-computer interaction; it seeks to understand how human values (e.g., welfare, accountability, autonomy, freedom from bias) can be accounted for in the design of computer technologies [6,7]. While Augmented Intelligence brought us the promising prospect to use the power of machine learning to uncover creative insights in fields such as science and healthcare, it's important for us to consider and incorporate human values in the design of AI systems. Value sensitive design framework has been proposed in the ethical considerations of micro-task crowdsourcing platform design [8]. Likewise, we should not underestimate the potential ethical concerns arising in the adoption and use of AI for intelligence augmentation. Researchers should continue to study how to avoid the risk of constructing machine intelligence that mirrors a narrow and privileged vision of society with its biases and stereotypes.

\section{Summary of Articles}

This mini-track presents two papers in HICCS-54. We introduce them briefly below.

Li, Xie, and Sha [9] present their novel approach of augmenting human creativity in product shape design by using the deep generative network. Their approach has two modules. The first module is a generative design module that generates part-aware $3 \mathrm{D}$ objects. The second module is a low-fidelity evaluation module that rapidly assesses the aerodynamic performance of the design. They adopt their locally linear embedding (LLE) to achieve the real-time performance evaluation. They demonstrate how their approach can be applied to car design to obtain the optimal aerodynamic performance.

Cerqueira, Althoff, Almeida, and Canedo [10] present a study that aims to generate an overview of the current state of the literature and software projects on AI ethics. They collect information about 182 research papers on AI ethics and 21 software projects from the GitHub for implementing AI ethics. Using the co-citation map analysis, they identify 15 clusters and five classes in the scientific literature of AI ethics. They use the text mining approach to analyze the 21 software projects for implement AI ethics and they find some interesting patterns.

\section{Reference}

[1] Russell, S. J., Norvig, P., Canny, J. F., Malik, J. M., \& Edwards, D. D. Artificial intelligence: a modern approach, Vol. 2, No. 9. Upper Saddle River: Prentice hall, 2003.

[2] Inhi Su, Keynote speech at HICSS 51st, 2018 January

[3] AGFA, "Augmented intelligence: The next frontier in health imaging", Healthcare IT News, 2018. [Online]. Available: https://www.healthcareitnews .com/news/augmented-intelligence-next-frontierhealth-imaging. [Accessed: 10- Oct- 2018].

[4] IBM, "IBM Cognitive Music", IBM Watson x Spotify, 2018. [Online]. Available: https://www.ibm.com /watson/music/. [Accessed: 10- Oct- 2018].

[5] Y. Li and K. Joshi, "The state of social computing research: A literature review and synthesis using the latent semantic analysis approach", in AMCIS 2012 Proceedings, 2012.

[6] B. Friedman, "Value-sensitive design", interactions, vol. 3, no. 6, pp. 16-23, 1996.

[7] Friedman, B., and Kahn Jr., P. H. "Human Values, Ethics, and Design," in The Human-Computer Interaction Handbook: Fundamentals, Evolving Technologies and Emerging Applications, J. A. Jacko and A. Sears (eds.), Mahwah, NJ: Lawrence Erlbaum Associates, Inc., pp. 1177-1201, 2003.

[8] X. Deng, K. Joshi and R. Galliers, "The Duality of Empowerment and Marginalization in Microtask Crowdsourcing: Giving Voice to the Less Powerful Through Value Sensitive Design", MIS Quarterly, vol. 40, no. 2, pp. 279-302, 2016.

[9] Li, X., C. Xie, \& Z. Sha. (2021, January). "PartAware Product Design Agent Using Deep Generative Network and Local Linear Embedding," in Proceedings of the 54th Hawaii International Conference on System Sciences.

[10] Cerqueira, Althoff, Almeida, \& Canedo.(2021, January). "Ethical Perspectives in AI: A Two-folded Exploratory Study from Literature and Active Development Projects," in Proceedings of the 54th Hawaii International Conference on System Sciences. 